\title{
Information Content Driven Model for Virtual Engineering Space
}

\section{László Horváth, Imre J. Rudas}

Óbuda University, Institute of Applied Mathematics and University Research and Innovation Centre, Bécsi út 96/b, H-1034 Budapest, Hungary

horvath.laszlo@nik.uni-obuda.hu,rudas@uni-obuda.hu

\begin{abstract}
Engineering practice is increasingly relied upon virtual technologies. Recent virtual solutions ensure integration of all engineering activities using system level computer representation of complex multidisciplinary engineering product. The authors have provided research results in developing strategies for leading engineering virtual technology, for more than twenty years. They analyzed paradigm shifts on the way to present virtual engineering systems and organized their work around their formerly published concept of Virtual Engineering Space (VES). These were preliminaries of the reported work in this paper. New method is introduced for structuring and representation of active driving knowledge content background $(K C B)$ in engineering model. The purpose of $K C B$ is to drive entity generation in the $K C B$ extended industrial engineering model of a product system. This paper introduces handling contextual initiatives and the application of them to define KCB structure for the generation of self-adaptive generic objects explaining role and structure of information content, as well as, the related model driving mechanism. Appropriately organized and configured engineering models offer a means for integration of engineering model based industrial, research and higher education applications in common VES. Recent industrial professional engineering modeling systems offer capabilities for implementation of KCB and application relevant configuration, of modeling capabilities for disciplines and human roles.
\end{abstract}

Keywords: virtual engineering space; system level product model; self-adaptive product object model; knowledge content background; multipurpose virtual engineering space

\section{Introduction}

Substantial changes in industrial products were followed by the dynamic development of virtual engineering methodologies and technology, over the previous decades. Development history of engineering modeling shows the way from the initial shape modeling to representation of generic industrial product system in virtual engineering space (VES). VES was a formerly published concept at the Laboratory of Intelligent Engineering Systems. The VES concept utilized 
the product model principle, as it was standardized in ISO 13303 [1]. Concept VES was developed from the concept of intelligent virtual product space (IVPS) [8] The above laboratory was founded at the John von Neumann Faculty of Informatics, Óbuda University for research and higher education in VES relevant issues in 2005.

According to its definition, VES represents product as contextual segment of the physical world [8]. The VES represented product behaves similarly in virtual, as it will behave in the physical world, after its production and installation. Beyond product structure, VES includes objects for production, marketing, and application of the product. During its manufacturing, advanced product is converted from virtual (model) system to cyber-physical system (CPS). CPS is composed of cooperating physical and cyber units and it is highly contextual with VES.

Systems based architecture of multidisciplinary products essentially changed the engineering practice toward application of virtual technologies at all activities during the whole lifecycle of the product from the initial concept to the recycling. For this purpose, leading engineering systems organized engineering activities around complex model of multidisciplinary CPS product. Beyond development, production, marketing, and application of product this model could serve the whole innovation cycle including fundamental, general problem solving, and product related research. In this way, theory and practice were integrated in VES. Main advantages of engineering activity concentration around central model for the whole innovation cycle are realization of all contexts, real time propagation of new findings through existing results, and quick reaction to new theories and methods at practice intensive industrial companies. Proactive product support can be provided for engineers among others to prevent inappropriate or not allowed object parameters and repetition of obvious errors from the past.

New discipline areas, such as, systems engineering (SE), requirements engineering (RE), system of systems engineering (SoSE), and applied informatics for high abstraction engineering modeling were enforced by system based products to integrate in engineering modeling. New objectives established consistent contextual structure in the product model. This placed new emphasis on application specific but, highly scientific and advanced mathematics. The need for modeling of the product operated by cooperating systems it also placed new emphasis on a wide-ranging structure of contexts. Context structure acts as glue in the product model. Product wide contextual modeling is considered one of the main achievements in early $21^{\text {st }}$ Century engineering systems.

Suddenly increasing complexity of engineering models resulted in a decreasing role of visualization by computer graphics. While human interaction with graphic visualization, in viewport, including dynamic navigation was still essential, emphasis was shifted to seeing and thinking in information space. This was inevitable because visualization capabilities of computer graphics proved to be inadequate for the information boom and deep knowledge. In this way, advances 
in engineering modeling replaced the emphasis on human capability for high level abstraction to conceptualize, develop, survey and understand complex model systems.

Because connected cooperating systems typically come from different discipline areas, in the same product structure, contextually connected mechanical, electrical, electronic, hardware and software units must be engineered in the same product model system. It is foreseeable that application of conventional engineering model systems, which represent different discipline related units in separated or not contextually integrated models, will be over soon at leading industries. Inevitably, future engineering descriptions and representations will be organized in contextual multidisciplinary models [15]. It will be essential to apply and reuse active knowledge for driving multidisciplinary engineering model entities in consistent context system.

Recognizing the above, strong trend of development, the authors of this paper started research in relevant issues more than 20 years ago. They organized work around their VES concept. One of main contributions in this paper is a novel method to establish structure and representation of active driving knowledge content background (KCB) to support generation of engineering model entities. A survey of paradigm shifts and our own related results in engineering modeling are discussed as preliminaries of the reported work. Objectives and scenario of the reported research are outlined and a KCB extended industrial engineering model is introduced. Following this, organizing contextual engineering models into VES, the role of KCB structure in self-adaptive object generation, human and contextual contributions and model driving mechanism are explained. Another main contribution in this paper is the integration of engineering models for industrial, research, higher education activities in complex VES and possibilities for implementation of results in industrial professional engineering modeling system are proposed.

\section{Paradigm Shifts and Preliminaries}

Research in this paper is part of a long-term program. Beyond the main research, this program includes continuous recognition and characterization of paradigm shifts. Leading engineering modeling strategies, principles, methodologies and systemics in current engineering modeling systems have evolved through these paradigm shifts over the past decades. Therefore, paradigm shifts, and our own related research results are surveyed for previous decades in Fig. 1. Similarly to other areas of science and technology, current engineering modeling relies heavily on former achievements. Most of the content of former paradigm shifts can be recognized in current engineering modeling and CPS systems. However, it is also 
important to recognize how things changed in the model environment of entities which contain former results.

Developments in factory automation needed geometric descriptions to generate programs for equipment and device control from the 70s. Typical results included symbolic descriptive language, geometric processing, and application related processing. Work in [19] can be considered as one of initial efforts in this area. Modeling of man-machine processes, served better communication between human and modeling procedures for better shape driven control [20].

The next paradigm shift introduced computer definition of engineering processes between the 70s and 90s. One of most advanced engineering process definition strategies was mixed application of variant and generative principles in the same solution [21]. Highly based on the result in [21], process and resource models were developed using vario-generative principle in the GLEDA system [22]. These models were extended to Quality Assurance [23] applications. One of the solutions for the connection of process and resource models with computer control of complex machine tools was introduced in [33] and an alternate method was applied in process plan definition and engineering system in [35].

The need for an engineer-understandable shape model was fulfilled by parametric solid form features in the 90s. Form feature modifies the previous shape and completes boundary representation of shape accordingly. Boundary representation (B-rep) is the prevailing geometric model which includes representations of surfaces and their intersection lines in boundary and places these geometric entities in Eulerian topology structure. The PRODES team joined early research in form feature based modeling and developed prototype modeling system in the $90 \mathrm{~s}$ [30]. Advanced ideas and methodologies were applied at development of this system including object oriented form feature driven model and integration of form feature and manufacturing models. Our research was in associative modeling of industrial processes considering solid form features [24]. Shape modeling still relies upon boundary represented geometric and functional form features. Form feature based modeling supports advanced modeling concepts such as dynamic product reconfiguration [18].

One of the main contributions to engineering modeling was the integrated product information model (IPIM, ISO13303) [1] which provided a means for integration of formerly separated partial models using generic and application resources, application protocols, and object-oriented scenario in the 90s. Analysis of object connections in this scenario resulted in the concept of integrated model object (IMO) [25]. Work for IMO also emphasized the importance of communication between human and modeling process.

Dramatically increased complexity of product structures demanded selfmodification product models capability of handling the propagation of extensive and frequent model changes during development, improving, correction, and variant creation activities. 


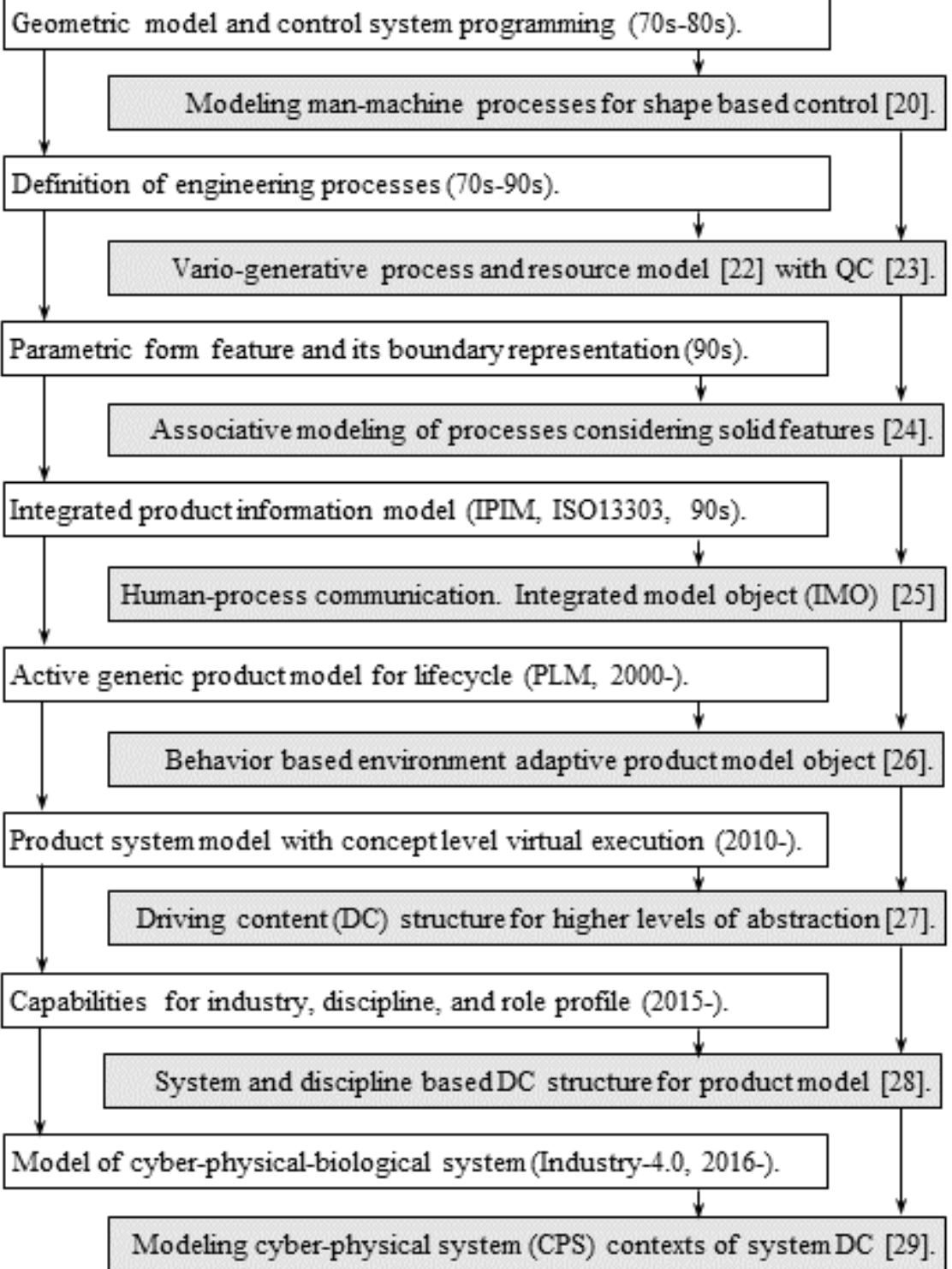

Figure 1

Paradigm shifts and relevant own research results in engineering modeling

An Active Knowledge Driven Contextual Generic Model was established for the above purpose at the beginning of the new century. Engineering activities served the entire lifecycle of product (PLM) [32]. As contribution to results in this area, 
our own research aimed establishing behavior based environment adaptive product model object [26].

The spread of systems cooperating in product structures required system level product model. The requirements, functional, logical, and physical (RFLP) structure was implemented from the systems engineering (SE) for this purpose [2] around year 2010. As a main advancement, the product concept was modeled on functional and logical levels together with behavior representation allowing virtual execution of product concept model. Our research targeted joining to the work in system level engineering modeling by contributions such as multilevel abstraction and concept of information content. These contributions supported establishing driving content (DC) structure on higher levels of abstraction [27].

Establishing engineering modeling for multidisciplinary system based products required flexible configuration of capabilities available at engineering workstations. To fulfill this request, leading engineering systems include configuration capabilities for industry, discipline, and role profile from around 2015. Our result is system and discipline organized DC structure for system based product model [28].

Currently, actual paradigm shift is in engineering modeling for cyber-physicalbiological system and Industry-4.0. Industry 4.0 means $4^{\text {th }}$ generation of automation using CPS and its information technology background. Key elements of this information technology are Internet of things, cloud computing, and cognitive systems. Our work is concentrated in representation driving content (DC) structure for CPS [29].

Efforts are done to organize company and institutional knowledge in intellectual property (IP). Need for high level content exchange between organized IP and DC structures is anticipated for the future. For that reason, paper [29] proposed multilevel structured IP entity which was devoted to transfer IP content between IP and DC structures. It was considered that IP organizations also serve applications other than engineering modeling.

\section{Objectives and Scenario}

In the long-term personal research program, main issues relevant to results in this paper are survey of model based engineering problem solving strategies [6], integration of product model objects [7], concept of virtual engineering space (VES) [8], modeling engineering objects on high abstraction levels [9], representation of engineer intent [10], development of driving information content structures [11], and multipurpose virtual space for engineering [12]. Objectives of research in this paper are developing improved version of former [11] information content based model driving, placing information content in the VES concept, 
defining the main driving context structure and driving mechanism, and integrating multiple applications in single VES.

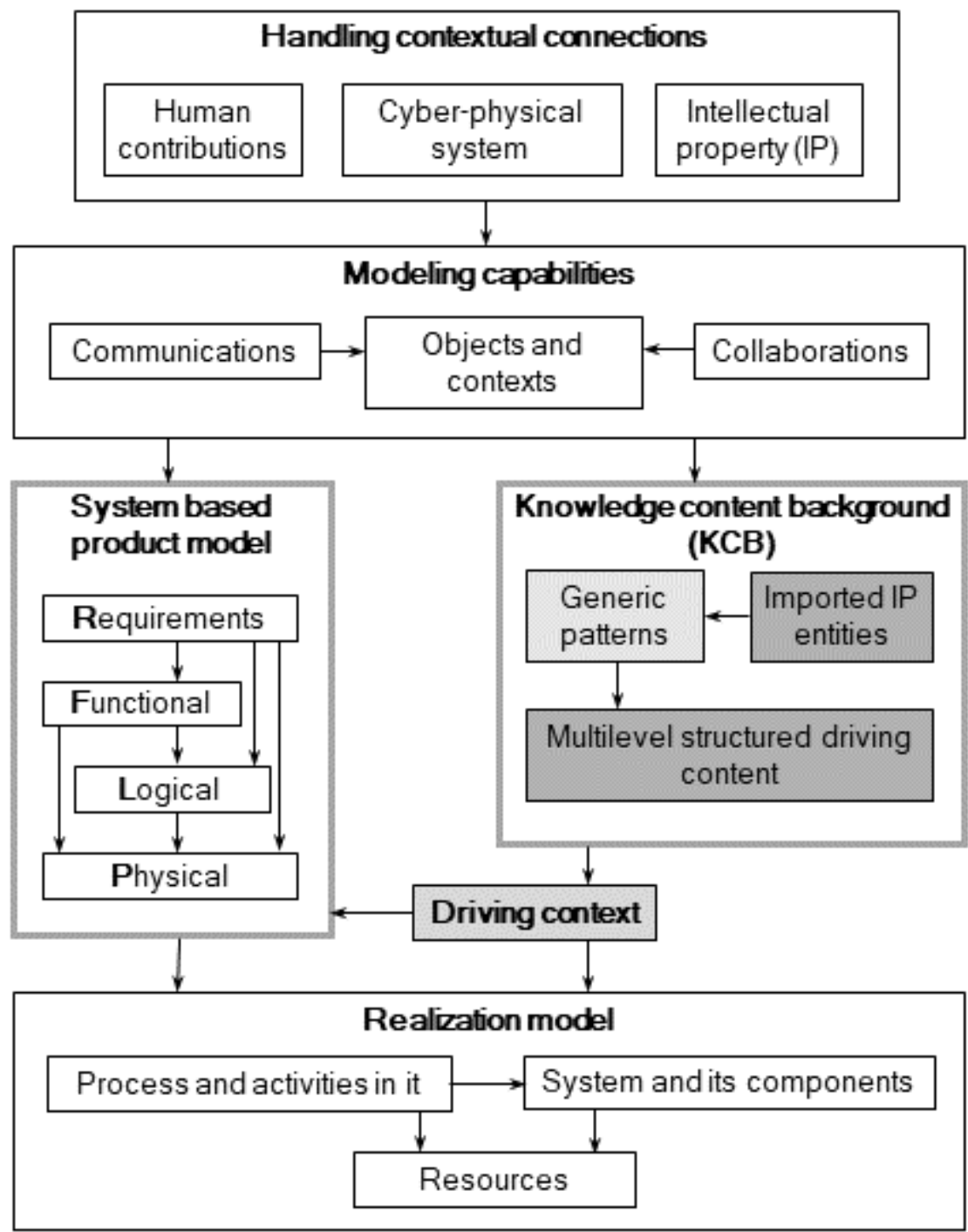

Figure 2

Scenario of modeling and subject of research

The main contributions of the research in this paper are the contextual structure of Knowledge Content Background (KCB); driving contexts from $\mathrm{KCB}$ structure elements to RFLP structured system level product model components, and integrated applications in VES. Term driving here means generation product 
model object parameters under control from appropriately contextual $\mathrm{KCB}$ object parameters.

Modeling multidisciplinary product needs the development of a functional and logical level concept model, before the related physically visible objects are developed. This is due to the complex structure of components from various disciplinary areas and their relationships would be hard or even impossible to handle only in a physical level model. Complex contextual structure of product components in a physical level model is best defined under driving from logical model. In this way, a physical model is generated by execution of a logical model. At the same time, definition of logical model components and their connections demands functional product components which are connected in our structure. Functional and logical level models are virtually executable when behavior representations are available in their components [13]. Definition of behavior representation is often done using advanced software, which is outside of the actual RFLP structure enabled engineering system. An example for this solution is the definition of dynamic behavior of a heterogeneous physical system using the Modelica open object-oriented modeling language [34].

There are frequent changing requirements against the product demanded description of these requirements within product model. This is served by first level in the RFLP structure in current solutions. The requirements (R) level specifies requirements the product must fulfill and relies upon the dynamically developed area of requirements engineering (RE) [16]. Functions (F) level is functional decomposition of the product to fulfill requirements. On the logical (L) level components include information for how functions are achieved. Finally, physical (P) level is result of execution the logical model. P level is purposeful structure of real world product features. RFLP structure is well proven methodology in systems engineering (SE) and uses core elements from the IEEE/1220 Standard for Application and Management of the Systems Engineering Process.

KCB is contextual extension of the RFLP structured industrial product model. It consists of imported intellectual property, generic patterns for driving content, and structured driving content (Fig. 2). The driving content structure consists of four levels and substructures on each level. This structure and its contextual connections will be introduced in section 5 of this paper. Relevant entities from imported IP and generic patterns are included in the actual VES or cited from outside as context. They also can be collected in VES for future reuse. Generic pattern organizes a set of closely connected essential contexts. These contexts are mandatory to apply and assist to avoid erroneous content and context definitions. Generic patterns are developed with developing ideas, experiences, and expertise at companies and other organizations.

Definition, generation, and application of RFLP and KCB structure entities require appropriately configured sets of modeling capabilities. Capabilities are 
considered as available at host engineering modeling system which is extended by $\mathrm{KCB}$ structure. Modeling capabilities are available for objects and contexts, as well as communication and collaboration processes.

The outside contextual connections of a product model system include human contributions, cyber-physical system (CPS) communications, and intellectual property (IP) import and export (Fig. 2). Human contributors are not only engineers on duty for actual engineering tasks but also other decision makers at application of standards, higher level decisions, law related affairs, etc. Advanced products can increasingly be regarded as CPSs [3]. The conventional connections with control units are being changed for increasingly smart communication between virtual and physical worlds. Cyber units in finished products can communicate with relevant VES representations for optimal operation and effective proactive malfunction handling. Intellectual property (IP) is established at a company or other organization not only to collect knowledge but also to make owning information clear.

Because a self-adaptive characteristic of the generic product model is relied upon, chains of contextual object parameters, context definitions have an exceptional role in VES [17]. Any object parameter has potential for participation, in one or more contexts. The engineering object parameter is modified only through a contextual chain, except for allowed exceptional manual interventions. The context is defined in object parameters or in the form of an individual object. Beyond its main content, context object includes time, variant, situation and event controlled activity parameters.

The realization model represents production related information. In recent advanced modeling systems, it has a three-level structure. Levels are for included manufacturing process and operational activities, the production system and its components and needed resources for realization. Recently, production systems increasingly adapt to CPS, in the scope of new paradigm Industry-4.0.

\section{Organizing Engineering Models into Virtual Space}

The next issue is how the complex product model in Fig. 2, can fulfill the criteria of virtual engineering space (VES). According to its definition in [8] VES represents a well-defined existing or planned segment of physical world together with its connections to all of the influencing outside virtual, physical and CPS environments. In engineering, the represented segment is a product, a prototype or an experimental object structure. The development process of VES includes model definition and integration activities to achieve the engineering requirements. The Model based Product Lifecycle Management (PLM) system [32] paved the way to VES level modeling. Unlike the previous product data management (PDM) 
system, this PLM system handles product information in a set of contextual models. Areas of contribution to VES, in this paper, are shown as shaded boxes in Fig. 2 and Fig. 3.

VES is developed during controlled contributions from responsible engineers, other persons and outside contexts (Fig. 3). Contribution from other persons and outside contexts are reviewed then accepted or rejected by responsible engineers except for items which are already decided and thus enforced by the VES or its contextual environment. In this way, a quick reaction of VES to a changed specification, rule, legislation, standard and other attempts for contribution, is critical. Outside contexts must represent connections with trusted information sources. Dedicated VES procedures review contribution attempts from all of the three sources for breaking existing accepted results and content, as well as, feasibility. The accepted contribution is handled as an initiative and is applied at the generation of the KCB extension or direct generation of generic product model entities (Fig. 3). An element in KCB extension drives relevant generic product model entities. Consequences of new accepted and validated contexts are automatically enforced through the self-adaptive mechanism of VES. Any accepted interaction from outside is propagated in the model along chains of contexts while organized active simulations save consistency, suitability, and quality of the model. One of important applications of self-adaptive generic model is dynamic reconfiguration of product [18].

The main challenge is a stable tradeoff between the acceptance/rejection reaction of existing model and revision of the formerly accepted results or content when necessary. Although preference information can be correct in the KCB structure elements, handling these items often requires human interaction, in accordance with local engineering decision rules. Generic patterns include methods for handling argued cases as necessary. Anyway, reliable measures are necessary to protect correctness and consistency of models. It is obvious that the participants of the engineering project, who are eligible, authorized and responsible for contribution in an actual VES, try to enforce their intent, utilizing personal excellence.

The component model of the product model is created in its own model space to represent part, assembly, analysis, simulation, etc. The model in this space represents generic engineering objects and contexts of their parameters. The model which is defined in its' model space is in the possession of self-instancing capability and is contextually connected to related models in VES. Beyond requirements, functional, logical, and physical component structures, simulation and model generation process structures can be included as component models in VES. Real-time operated complex simulations provide advanced support for VES activities. Model generation processes are contextual with object representations and descriptions and serve regeneration of models when it is necessary. The generic characteristic of the model objects is essential to provide a capability for self-modification and self-instancing. To achieve this, the product model must 
include proper active knowledge representation to generate modified or new entities.

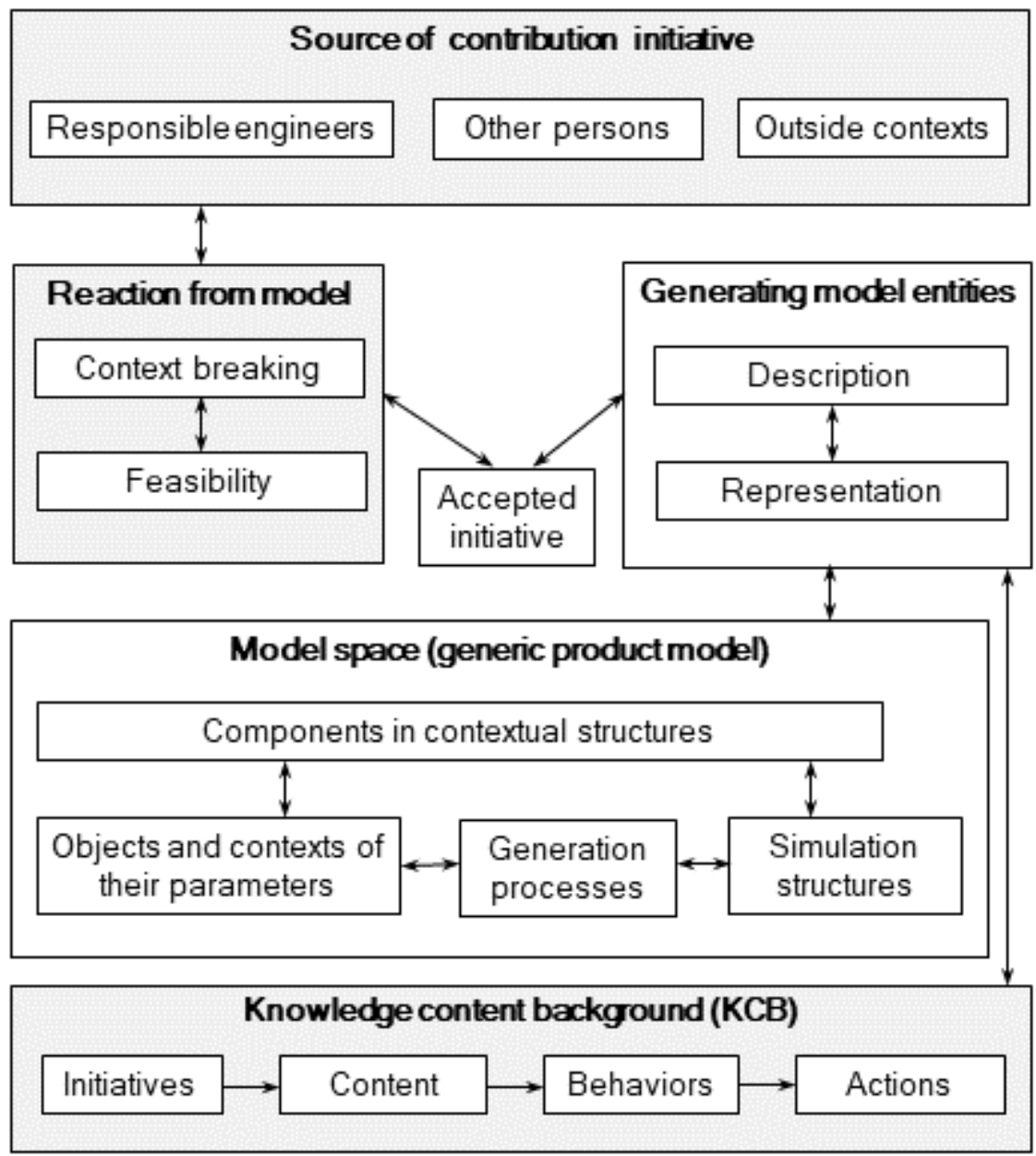

Figure 3

Contributions to VES

A four-level, product-wide, content element structure is proposed, for the knowledge content background (KCB) (Figs 3 and 4). Initiatives (I) level includes elements with personally represented information for accepted and processed contributions. Elements in the communicated content (C) level carry validated and accepted content. The acceptance process may result in an activated or deactivated element. A deactivated element is an option for later, changed circumstances. Behavior level (B) is an attempt to establish a product-wide contextual structure of the system and function-driven behavior content. 
The actions (A) level includes elements to the drive P level entities, in the RFLP structure. Future development of level A elements is planned to serve their twoway connection with cyber units in CPS system. Development towards smart companies makes this feature of $\mathrm{KCB}$ more important and at the same time feasible for the future.

As the global integrator for a well-organized engineering model structure, VES is art of science, technology, and engineering. The sophisticated product model has a great impact on the effectiveness of advanced engineering activities. VES requires human background for collecting content and establishing the model structure. $\mathrm{KCB}$ was intended to provide a modeling environment which supports seeing and thinking in VES. Measures to check initiatives by VES content management are very important to avoid context breaking, infeasible tasks, mistaken representation and inconsistent object representation.

\section{Information Content in Self-Adaptive Generic Driving Objects}

As an organic extension of the driving mechanism in RFLP structured product model system, active KCB drives the product model entities through chains of contextual object parameter connections using knowledge representation as content. The RFLP structured product model definition currently applies very complicated dialogues between model generation procedures and collaborating humans. This definition of complex model entities and their relationships, need various contexts to consider as normal, during interactive human dialogues in collaborative engineering process. This problem motivated research, in raising human interactions to a higher-level abstraction, where better survey was provided and less contexts should be kept in mind during model definition. As result of this research, information content based driving [9] was conceptualized to replace dialogue definitions. Ackoff [35] classified content of the human mind into data, information, knowledge, understanding, and wisdom categories. Research in content kept this categorization in mind at analysis of higher level abstraction.

The Information content (or simply "The Content") describes and represents all information and knowledge in the background of coordinated decisions on information in generic product model entity (Fig. 3) parameters. Driving new and modified entities in RFLP structured model by active content provides new capability for the definition of consistent product model. In this way, driving by content can gradually replace complex dialogue definition of entities. Active content can flexibly represent and organize theoretical considerations, research findings, methodologies, experience, expertise, and intuition. 
Increased complexity places high emphasis on consistency of model during the whole lifecycle of product. Product malfunctions are often caused by neglected or misleading context. To handle this problem, concept contextual consistency was included by the authors. A contextually consistent model includes proper set of organized contexts to ensure correct consideration of all influencing factors. Organized context set is revised during lifecycle of product when new context arises or existing content is abandoned at analysis, simulation, or physical product repair.

Shape representation applies concept topological consistency for decades. Consistent topology is assured by geometric modeling in engineering systems. Leaving assurance of topological consistency as separately handled in geometric modeling, topology of shape is not part of $\mathrm{KCB}$ context structure. At the same time, contexts of other model entities in RFLP and KCB structures with vertex, edge, and face topological entities must be included. Analysis and assurance of contextual consistency is planned as issue of future research at the Laboratory of Intelligent Engineering Systems. Thus, they are not issues in this paper.

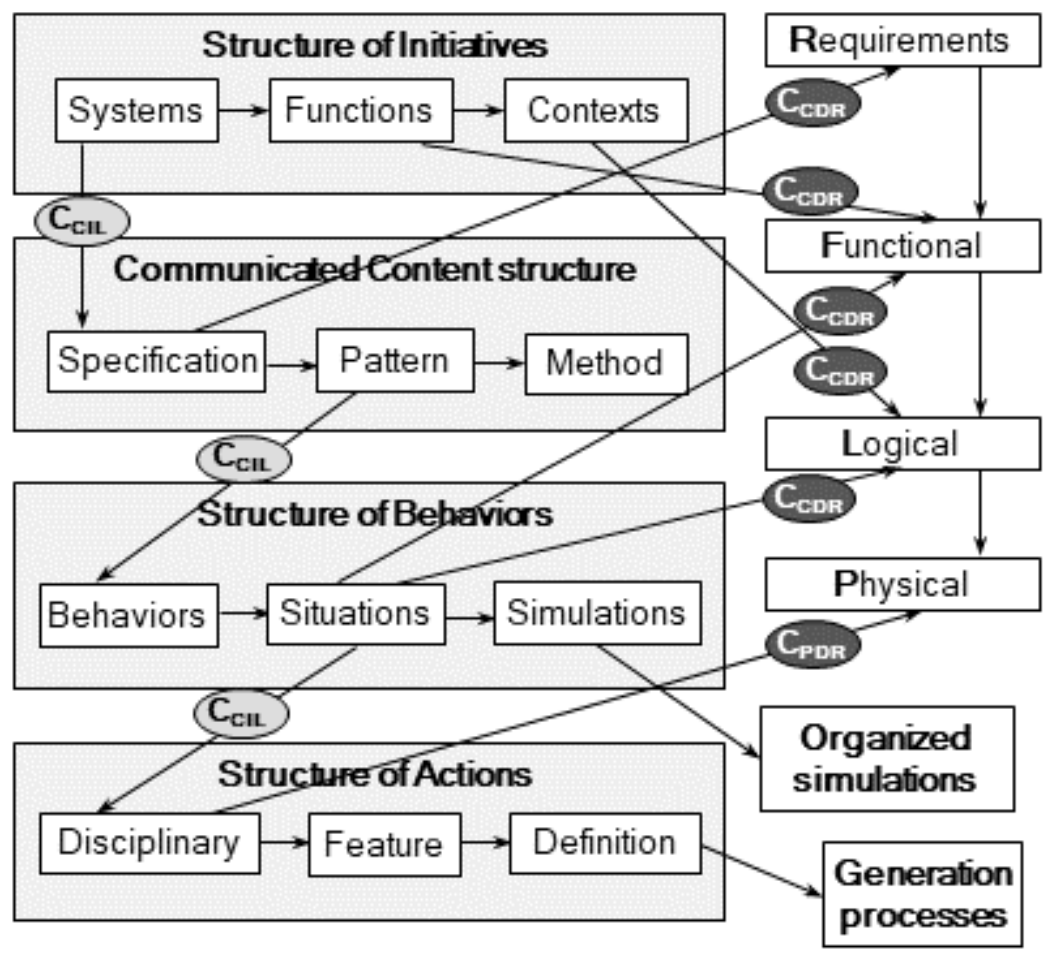

Figure 4

Main RFLP and KCB contexts 
KCB level consists of contextually connected thematic substructures (Fig. 4) while substructure consists of contextually connected elements. KCB element drives one or more elements and components in KCB and RFLP structures, respectively. $\mathrm{KCB}$ elements and their connections are free to define under constraining by relevant active driving contextual definitions in the model. To ensure global consistency of model, some contextual connections (or simply contexts) are mandatory to include. Fig. 4 shows the set of mandatory contexts. These contexts form the main structure of model. They are inter-level context $\left(\mathrm{C}_{\mathrm{CIL}}\right)$ between elements on different levels of $\mathrm{KCB}$ structure, concept driving context $\left(\mathrm{C}_{\mathrm{CDR}}\right)$ between $\mathrm{KCB}$ element and $\mathrm{R}, \mathrm{F}$, or $\mathrm{L}$ level component, and physical driving context $\left(\mathrm{C}_{\mathrm{PDR}}\right)$ between $\mathrm{KCB}$ element and $\mathrm{P}$ level component. Physical driving context was distinguished in KCB for applications where only the physical level exists in the product model, in the case of not RFLP enabled modeling system or model, which does not need concept level representation.

Although result of an engineering activity highly depends on the creativity of responsible engineers, contextual active knowledge items, represented in pattern and content entities, restrict role relevant human initiatives to avoid incomplete, erroneous, or contradictory representation. In default, $\mathrm{KCB}$ does not accept any contribution which contradicts any previously accepted active item. At the same time, any new contribution can create a debate result of which may be change or abandon formerly accepted KCB item. This evolution of KCB and RFLP model is essential during the whole lifecycle of product. Broken but abandoned higher level context is deactivated or deleted. This mechanism is enough flexible to accommodate situation dependent decisions on object parameters.

Initiatives are recorded and organized to accommodate and relate human intent in collaborative environment. Initiative (I) level substructures in the KCB structure organize systems, functions, and contexts for a generic product. Content in initiative is referred in elements of these substructures. "I" level makes discipline independent initiative definition possible. The systems-functions-context substructure chain allows for representation of main background content demanded by recent system-organized product structure.

The purpose of communicated content (C) level is to organize specifications which are active for the systems. Patterns and methods are organized for specification. Beyond the main contexts between systems and specification substructure elements, contexts are defined between elements in the rest substructures on "I" and "C" levels as required to transfer referred content. Consistent structure is checked for driving contexts. Context may also have a checking or an announcement status.

As it was emphasized previously, in this paper, behavior representations gained outstanding importance by their role at virtual execution of $\mathrm{F}$ and $\mathrm{L}$ level models in RFLP structure. It can be said that the representation of behavior brings life to the RFLP structured product concept model. Substructure chain on behaviors (B) 
level of KCB structure provides structure of behaviors, structure of situations for behaviors, and simulations for situations. The Situation Element organizes circumstances. Some of recent modeling systems have capabilities for representation organized simulations in product model. These simulation entities can be driven by elements in the simulation substructure (Fig. 4).

The fourth (A) level of KCB structure organizes content for physical level driving actions. Because physical level engineering is divided to disciplines, disciplinary substructure organizes discipline related blocks of physical level product representation. This method is sufficiently flexible to handle disciplinary combinations in the engineering practice. The other two substructures organize features and their definition methods for discipline substructure elements.

As it was defined in [6], physical level engineering object fulfill eligibility criteria for its representation as feature in a feature driven product model. The "A" level of $\mathrm{KCB}$ structure assumes availability of feature definition to any physical level engineering object in the host modeling system. Element in definition substructure includes content for driving generation procedures of relevant physical level features in the host modeling system. Element also can be defined to drive model generation process entities where these entities are included in product model to capture engineering processes as knowledge representing proven practice for instant and future application. This advanced function was established at leading engineering systems on the way to automate engineering modeling. Process entity often serves set of procedures needed to generate physical level model of a welldefined product unit. Cross-disciplinary contexts can be placed in feature and definition substructure elements to connect features from different disciplinary areas. Cross-disciplinary modeling on physical level is an actual research issue [14] and future work will include further analysis at the Laboratory of Intelligent Engineering Systems.

\section{Model Driving Mechanism}

As it can be concluded from the above explanations, driving of the object parameter generation is critical in the proposed contribution to active adaptive product modeling for lifecycle of product. On one hand, the model must have the capability to modify itself automatically, using active contexts. On the other hand, using an active context set, which is not appropriate for the actual situation, can cause serious malfunctions in the operation of the represented product. Moreover, CPS product includes cyber units, which are created in virtual and may have VES connections, for control of physical product processes on the basis of actual sensor network information. 
The organized driving of the RFLP model entity generation ensures consideration of all contexts using consistent context structure. The ad-hoc connection of the model object parameters is replaced by organized driving content structure. This means that object parameter is defined in the context of relevant parameters of relevant objects within the KCB structure or between KCB and RFLP structures. Context relevancy is important to make map for consistency analysis. However, it is impossible to prepare all driving contexts for automatic action along context chains. Regardless, responsible engineers must have role based means to sight into the modeling process at critical points. For the above purposes, role based, human interaction contexts are defined. When consistency analysis reveals a lack of context, a void context is generated and role based intervention is enforced by the modeling system. Driving RFLP structure components is like host modeling, system specific. Consequently, specialties of the connected RFLP structure must be considered at implementation of the KCB structure and its driving mechanism.

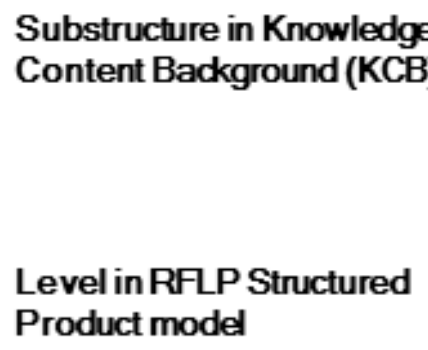

Level in RFLP Structured product model

Substructure in Knowledge Content Badkground (KCB)

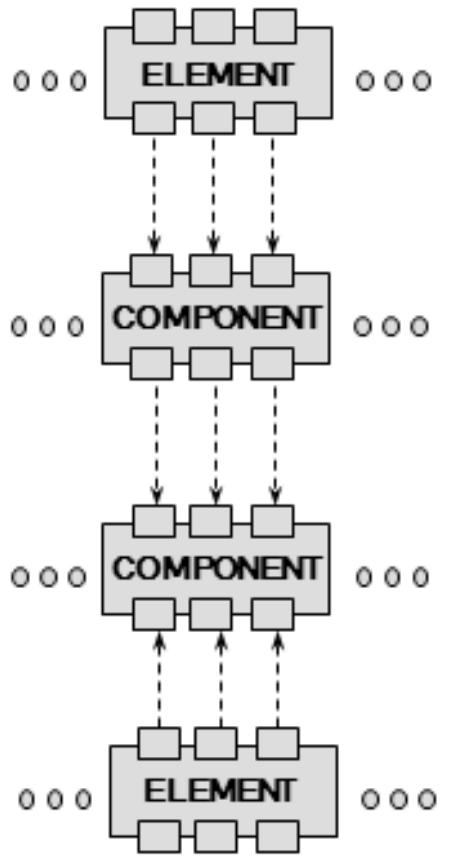

Figure 5

Communication through ports

Structural similarity between RFLP and KCB offers the benefit of application the same model construction methods and procedures. Consequently, KCB structure applies the same procedures and formats as management of RFLP structure in the host engineering system for the connection of model entities. Therefore, driving procedures and formats are RFLP modeling dependent. Known RFLP structure management systems apply ports on the component for communication with other 
components. Ports are free to define, as demanded by the model under development. Port based connection is illustrated for two connected RFLP structure components and their KCB driving elements in Fig. 5. Elements and components in Fig. 5 apply three ports to establish connection between two entities, exchange information along this connection, and control of the connected element or component. A generic model is highly based on situation driven activity of elements and components using control port connections.

\section{VES Integrates Application Areas}

Developing strategies in engineering modeling highly relies upon four key concepts namely, integration, information space, multidisciplinary characteristic, and systems. Integration was continuous intention during long-term history of engineering modeling development. It was motivated by the need to replace manual and exchange types of data communication between separated units, objects and procedures by direct connection in computer based engineering systems. In this way, the integrating capabilities of models were extended continuously.

Information space accommodates, organizes and coordinates engineering resource originated information for the lifecycle of the product. It is an important concept for integration. In its generalized definition, the product is an organized structure of engineering objects. Engineering objects were analyzed and characterized in [8]. The main resources for information are human capabilities, modeling capabilities, control units, physical units, engineering objects, methodologies, processes, intellectual property and information handling related capabilities. Recently, information and other resources are available from the cloud as a service. The conventional option of premise installed resources does not provide sufficient capabilities and flexibility for VES installation.

The style, purpose, media and environment of engineering work has changed. This means there is a need for revision of classical organization, integration, methodology and activity in engineering. Spreading cyber-physical systems (CPSs) generated urgent need for extension of product and production modeling capabilities to multidisciplinary systems. CPS is inherently multidisciplinary, while industrial product and production systems are often handled as CPS, as their engineering. CPS requires integration of theory and practice within the same model and needs capabilities for fundamental, problem related and product related research, in the product modeling system [2].

Extensive involvement of industrial organizations in engineering related research forced the independent research organizations to consider integration of their industry related research projects with industrial projects. Separated modeling 
installations at cooperating research and industrial organizations cannot provide real integration and generate a need for parallel installations of extended modeling capabilities and CPS laboratory equipment at the research organization. Cloud serviced VES offers a new chance for deep integration, using high level contextual driving between related models within the same VES. VES may consist of full value contextual component VESs in cloud environment, where work at research organization is still independent. The product model can be configured among others for experiments and engineering problem analysis. The Cloud also provides connection with outside CPS installations, including Industry-4.0 eligible smart factories.

It was recognized that the above joint VES can be extended to higher education organizations, where effective connection with outside research, industrial, and higher education organizations was a problem area for decades [36]. The Industry4.0 paradigm shift and other changes have major impact on the requirements demanded of higher education programs in engineering. Undergraduate, Graduate, Postgraduate and Doctoral courses are forced to reorganize, for the new and changing issues, such as, multidisciplinary engineering, CPS, virtual space, advanced modeling; cloud serviced engineering and integrated theory/practice. It is not hard to anticipate that resources for changed programs can be provided only in virtual system in integration with cooperating industrial and research organizations. In virtual environment, effective industrial organization-higher education and research organization-higher education dual cooperation's can be configured and realized. High level and leading laboratory capabilities and capacities can be accessed as cloud based service from laboratory, experimental, or real industrial CPS systems.

The VES concept for multiple applications is summarized in Fig. 6 while Fig. 7 includes contextual information structures for industrial project, research program, and university course VES application types. Each application type uses an internal component-VES specific set of contextually integrated models within project organization in the host engineering modeling system. Component-VES applies type specific strategies, methods, communication, and representations. Component VESs are connected by driving contexts as demanded. These contexts can be applied at configuring "virtual" component-VES connecting units from different VES types. Modeling capabilities are configured for type specific profile of component-VES considering relevant disciplines and human roles.

The concept of VES, stand alone or component, can be followed in Fig. 6. VES objects are generated in the context of initiatives. Sources of initiative contexts are human interaction and trusted outside object connection. Initiative is contribution to define VES configuration, strategy, specification, and communication. The configuration is defined for application profile including application areas, disciplinary areas and roles and for capabilities in accordance with application profile. Definition of configuration drives the "work" in VES, to establish and develop configuration, handling of structures, product entities and communication. 
This work is done using the configured capabilities in the host engineering system and it provides results for the VES model. VES model consists of VES administration, virtual spaces (or component-VESs), model objects and generated outside contexts.

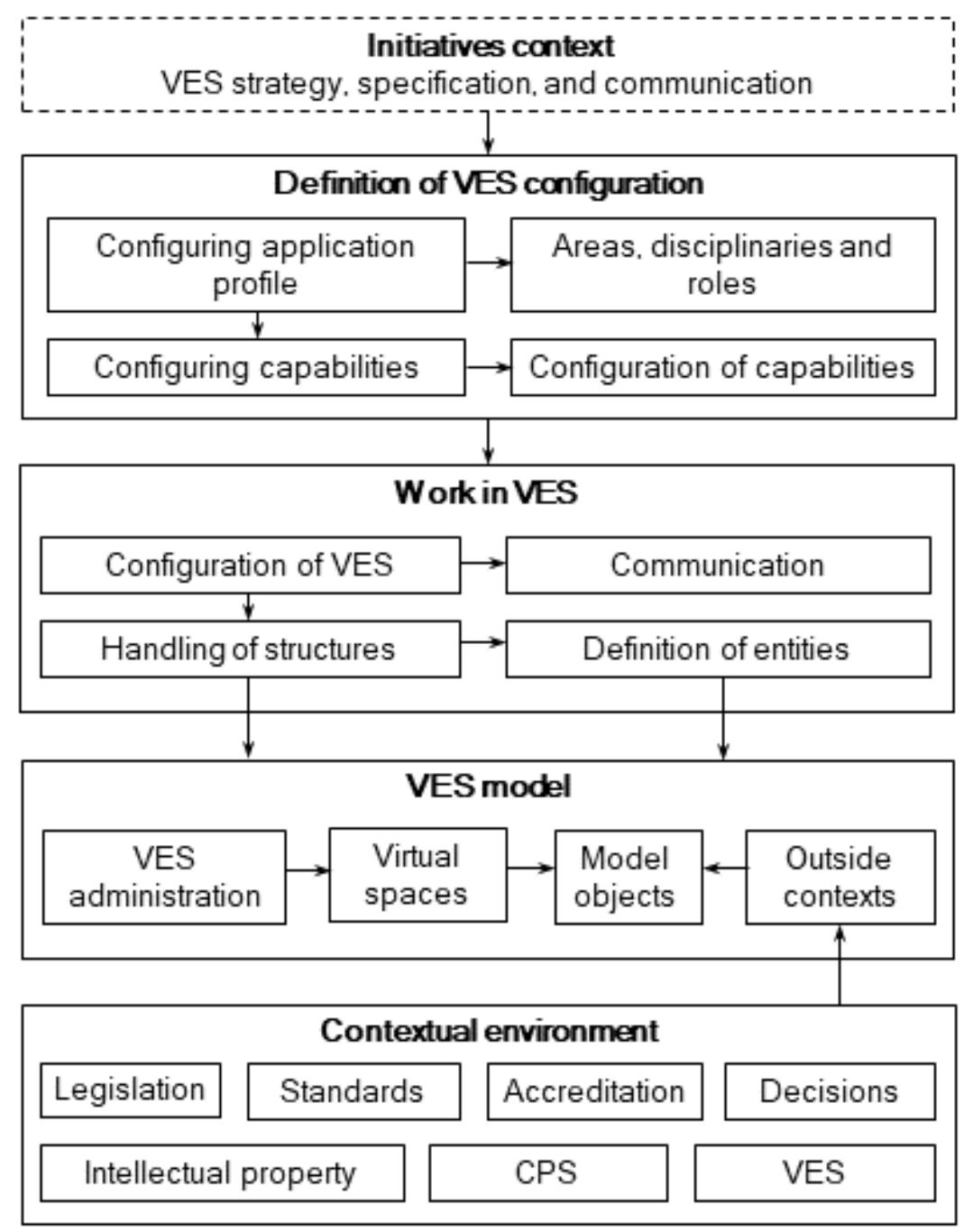

Figure 6

VES concept for multiple applications

While initial outside contexts are connections to control VES, generated outside contexts are connections which are decided in VES using recognized communication demand. VES entities are generated by relevant procedures in the host engineering modeling system. Because VES represents a separated segment 
(or part) of physical world with connections with other related segments of this world, its connection with contextual environment is critical. Main units in the contextual environment are legislation, standard, accreditation, higher level decision, intellectual property, CPS and other VESs. In this way, VES must be fitted organically into its environment.

The VES type specific contextual information structures which are represented for activities in industrial, research, and academic applications are shown in Fig. 7. This allows for more sight into the proposed multipurpose VES. Information structures in Fig. 7 are results of initial analysis for the case where VES is based on KCB driven RFLP structured product model. This means that information units in Fig. 7 must be represented in the KCB-RFLP model structure. As it was noted above, product model sometimes is restricted to pure physical level. Owner related information for inventions, patents and know-how must be strictly included for any outside contextual connection of VES. Recently, in addition to product development, prototyping and application, industrial application may serve processes and activities at an Industry-4.0 eligible smart factory.

Industrial engineering activities start with a problem definition and its solution, which are served by collaborating human and intellectual property driven initiatives. Initiatives drive the definition of the problem to be solved and the process of solving. Problem solving works and produces results at the concept, physical and prototyping levels. CPS connections are required for physically produced and tested prototypes, application-ready products and the relevant production system. CPS physical units are connected and operated by cyber units. Nevertheless, physical units also have physical connections. Former concept of a virtual company needs reconsideration and application in this changed environment. Cyber elements of CPS can be configured within VES and they can be serviced from VES in the cloud.

Engineering, research type of VES application generally serves industrial problem solving, directly for engineering at a company or indirectly to produce general results. As it was explained herein, research activity is within an industrial or research application type of VES. In a research type VES application, activities start with definition and generation of objectives. This activity is driven by collaborating humans as well as resources and results (R\&R). In this context, resources are utilized at research while results are referenced from work of others as initial conditions. Objectives, together with other initiatives, are applied at the generation of the research plan. Among others, initiatives drive principles and methods of research. Results of research are collected in experiments and findings. CPS connections are required for physical prototypes and laboratory installations.

Academic application serves higher education programs and includes specifics different from the other two applications. At the same time, the old problem is how to include industrial and research specifics in course programs. VES provides solution for this problem by offering an organic integration, of real industrial and 
research units, into higher education programs in highly integrated model. Course programs, the knowledge in them, case studies, drills, assignments, assessment, laboratory tasks and teaching materials can be realized in the form of specific object models in active model system. Activities start with competences, which are driven by a collaborating Teaching Expert and Executive Human and Course Control (CC) initiatives.
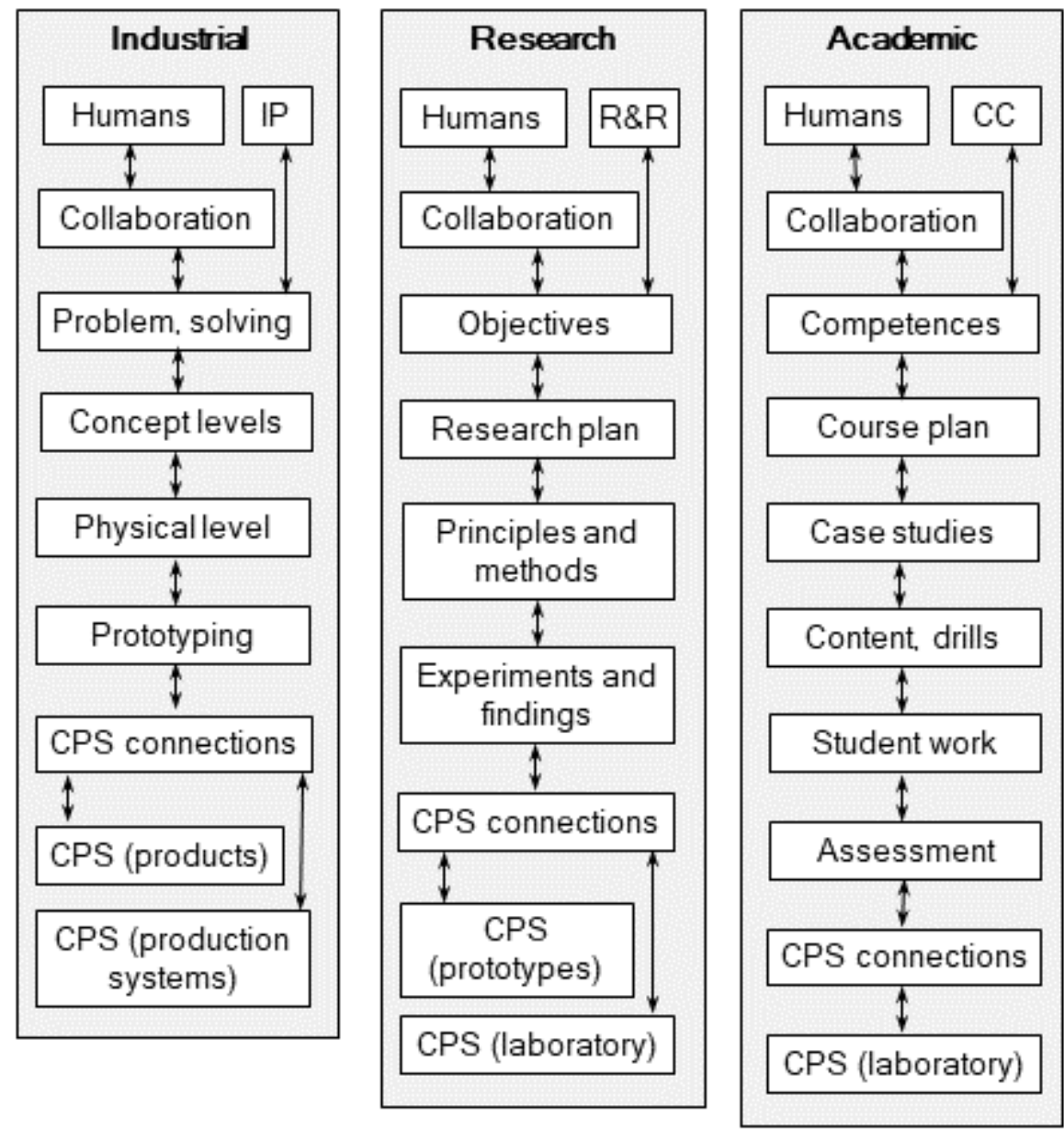

Figure 7

Information structures for VES applications

CC comes from, law, legislation, accreditation, teaching content, higher level decision and customer demand. In engineering education, the main customers are students and their potential employers. Initiatives and the resulted competences drive the course plan. Definition of competences must include information suitable for model generation. Course model can be considered as a very specific 
product model, in which contextual engineering objects are represented to understand their systemics, principles, operation, characteristics, parameters and relationships. Active engineering objects represent a configuration of explanations, experiments, drills, assignments and assessment. Main contextual units of course information include, case studies, content and related drills, student work and assessment. CPS connections are required for laboratory installations.

\section{Implementation Issues}

The proposed KCB structure and multipurpose VES models are planned to integrate in an appropriately configured host industrial engineering modeling system product, where resources are accessed from the cloud as a service. It can be stated, that current advanced engineering systems, offer the demanded communication, modeling, development and management resources. Operation of $\mathrm{KCB}$ as an organic part of the product model uses the capabilities for model construction and configuration of the modeling environment. KCB definition, generation, and integration tasks can be realized by new object classes and related new procedures. Object handling and access to user surfaces and model structures are available, as open system functions in the host modeling system product. In this way, KCB related modeling capabilities, model object structures and model system management can be tailored. User defined model structures, algorithms; mathematical functions, etc. are included in actual and future models. The Application Programming Interface (API) is available for procedures which can use existing resources in the host modeling system.

Implementation of VES for multiple applications in industrial modeling systems needs future research and development in objects, communications, structures and the drives for research and academic applications using pilot VES. These applications demand much more specific objects, than the KCB structure. Model structures at these applications are different from the well proven product model structures. The main challenge is to transfer administrative, visualization and data transfer-centric processes to the world of contextual model structures. An experimental cloud based engineering environment configuration, is planned for development of integrated VES application areas, at the Laboratory of Intelligent Engineering Systems in the future.

\section{Conclusions}

This paper introduces some of the latest research results, in the information content supported driving of RFLP structured product model entities and multipurpose VES configurations, at the Laboratory of Intelligent Engineering Systems at Óbuda University. The work was motivated by new demands in engineering modeling, for the representation of cooperating multidisciplinary 
systems, based on CPS products, self-adaptive generic knowledge driven contextual product models, highly integrated research, product development, manufacturing and application activities, development of product model towards virtual space and consideration of the new paradigm of Industry-4.0.

The above demands have brought new challenges for industrial engineering, engineering related research, operation of CPS installations and higher education in engineering. The study in the integration of model based activities required a historical survey of paradigm shifts and the relevant internal research results in engineering modeling in this paper. The main contributions of this paper are the updated scenario of engineering modeling, the new concept for organizing engineering models in VES including application oriented component-VES, the representation of information content as initiative based self-adaptive generic KCB driving structure and the information structures for application specific component-VESs. KCB driving content structure was conceptualized to fill the gap between contextual initiative sources and the RFLP structured product model.

Future research initiatives will include work on detailed driving structures, content entity structures, contextual consistency and integration of engineering models with Cyber Units of Cyber Physical Systems (CPS). The latter will be a contribution to CPS product related modeling for manufacturing, maintenance and optimized, malfunction-free operations.

\section{Acknowledgement}

The authors gratefully acknowledge the financial support by the Óbuda University.

\section{References}

[1] R. Jardim-Goncalves, N. Figay, A. Steiger-Garcao: Enabling interoperability of STEP Application Protocols at meta-data and knowledge level, International Journal of Technology Management, 36(4) pp. 402-421, 2006

[2] S. Kleiner, C. Kramer: Model Based Design with Systems Engineering Based on RFLP Using V6, in book Smart Product Engineering, Springer, pp. 93-102, 2013

[3] A Canedo, E Schwarzenbach, E. M A Al Faruque: Context-sensitive synthesis of executable functional models of cyber-physical systems, In 2013 ACM/IEEE International Conference on Cyber-Physical Systems (ICCPS), Philadelphia, PA, USA, pp. 99-108, 2013

[4] J. A. T. Machado, A. Babaei, B. P. Moghaddam: Highly Accurate Scheme for the Cauchy Problem of the Generalized Burgers-Huxley Equation, Acta Polytechnica Hungarica, 13(6), pp. 183-195, 2016 
[5] D. Wua, D. W. Rosena, L. Wangb, D. Schaefera: Cloud-based design and manufacturing: A new paradigm in digital manufacturing and design innovation" Computer-Aided Design, 59, pp. 1-14, 2015

[6] L. Horváth and I. J. Rudas, Modeling and Problem Solving Methods for Engineers, Elsevier, Academic Press, New York, 2004

[7] L Horváth: Towards Knowledge Driven Adaptive Product Representations, in book Advances in Soft Computing, Intelligent Robotics and Control. Heidelberg, London, New York, Springer, pp. 191-209, 2014

[8] L. Horváth: Supporting Lifecycle Management of Product Data by Organized Descriptions and Behavior Definitions of Engineering Objects, Journal of Advanced Computational Intelligence and Intelligent Informatics, 11(9), pp. 1107-1113, 2007

[9] L Horváth, I. J. Rudas: Multilevel Abstraction Based Self Control Method for Industrial PLM Model, 2014 IEEE International Conference on Industrial Technology, Busan, Korea, pp. 695-700, 2014

[10] L. Horváth and I. J. Rudas: Human Intent Representation in Knowledge Intensive Product Model, Journal of Computers, 4(9), pp. 954-961, 2009

[11] L Horváth: Towards Knowledge Driven Adaptive Product Representations, in Advances in book Soft Computing, Intelligent Robotics and Control. Springer, Heidelberg, London, New York, pp. 191-209, 2014

[12] L. Horváth, I. J Rudas: Role of Information Content in Multipurpose Virtual Engineering Space, IEEE $15^{\text {th }}$ International Symposium on Applied Machine Intelligence and Informatics, Herlany, Slovakia, pp. 99-104, 2017

[13] K. Baughey: Functional and Logical Structures: A Systems Engineering Approach" SAE 2011 World Congress, SAE Technical Paper 2011-010517,2011

[14] G. Beier, A. Figge, R. Müller, U. Rothenburg, R. Stark: Supporting Product Development through Cross-Discipline Dependency-Modeling, Novel Approaches for Traceability-Usage, Lecture Notes on Information Theory, 1(1), 21-28, 2013

[15] J Lefèvre, S Charles, M Bosch-Mauchand, B Eynard, É Padiolleau: Multidisciplinary modelling and simulation for mechatronic design, Journal of Design Research, 12(1-2), pp. 127-144, 2014

[16] A, Sutcliffe, S. Thew, P. Jarvis: Experience with user-centered requirements engineering, Requirements Engineering, 16(4), pp. 267-280, 2011

[17] A. Brière-Côté, L. Rivest, A. Desrochers: Adaptive generic product structure modelling for design reuse in engineer-to-order products, Computers in Industry, 61(1), pp. 53-65, 2010 
[18] J. Lee and D. Muthig: Feature-Oriented Analysis and Specification of Dynamic Product Reconfiguration, in book High Confidence Software Reuse in Large Systems, Springer Berlin, Heidelberg, pp. 154-165, 2008

[19] M. Berta, I. Cser, Computer-aided operation planning and programming of machining on turning centers, in Proc. of SPIE 3832, Sensors and Controls for Intelligent Machining and Manufacturing Mechatronics, pp. 1-6, 1999

[20] L. Horváth, I. J Rudas: Modeling Man-Machine Processes in CAD/CAM and Flexible Manufacturing Systems, in Proc. of the 1996 IEEE 22 $2^{\text {nd }}$ International Conference on Industrial Electronics, Control, and Instrumentation, Taipei, Taiwan, pp. 1484-1489, 1996

[21] M. Horváth, Semi-generative process planning for part manufacturing, in proc. of the International IFIP/IFAC Conference on Programming Research and Operations Logistics in Advanced Manufacturing Technology (Ann Arbor, Mich., USA), North-Holland Pub. Co., Amsterdam, New York, Paper MS 79-153, pp. 1-9, 1979

[22] L. Horváth, K. Szabó, GLEDA, A computer-aided Manufacturing Process Planning System for Parts to be Machined, in proc. of the APMSCOMPCONTROL' 85 conference, Budapest, pp. 618-629, 1985

[23] I Cser, Gy Hermann, L Horváth, PC-based quality system for manufacturing of mechanical parts, in proc. of the $4^{\text {th }}$ international conference on CAMP 94, CAD/CAM and multimedia, Budapest, Hungary, pp. 164-169, 1994

[24] L. Horváth, I. J Rudas, G, Hancke: Associative Modeling of Machining Processes Using Feature Based Solid Part Models, in Proc. of the $200026^{\text {th }}$ Annual Conference of the IEEE Industrial Electronics Society, Nagoya, Japan, pp. 1267-1273, 2000

[25] L. Horváth, I. J. Rudas: An Integrated Description for Intelligent Processing of Closely Related Engineering Objects, in Proc. of the 2006 IEEE International Conference on Systems, Man \& Cybernetics, Taipei, Taiwan, pp. 4886-4891, 2006

[26] L. Horváth, I. J. Rudas:Human Intent Description in Environment Adaptive Product Model Objects, Journal of Advanced Computational Intelligence and Intelligent Informatics, Tokyo, 9(4), pp. 415-422, 2005

[27] L. Horváth, I. J. Rudas: Filling the Gap between Human Thinking and Model Object Generation at Product Definition in Modeling Systems, in Proc. of the IEEE-ICIT 2010 International Conference on Industrial Technology, Viña del Mar, Chile, pp. 1012-1017, 2010 
[28] L. Horváth: Content for Context Structure in Multidisciplinary Engineering Model, in Proc. of the IEEE International Conference on Systems, Man, and Cybernetics Conference Budapest, Hungary, pp. 3824-3829, 2016

[29] L. Horváth: New Method for Definition of Organized Driving Chains in Industrial Product Model, in Proc. of the 2017 IEEE International Conference on Industrial Technology, Toronto, Canada, pp. 1183-1188, 2017

[30] I. Horváth, P. Kulcsár, Zs. Gábor, Z. Bagoly, A. Horváth, V. Thernesz: Implementation and Uniform Management of Modelling Entities in a Massively Feature-Object Oriented Advanced CAD Environment, in Periodica Polytechnica Ser. Mech. Eng., Vol. 39, No. 2, pp. 81-113, 1995

[31] T. Tóth, D. Vadász: TAUPROG-T: Programsystem for Automatic Planning of Complete Technological Process of Rotation Symmetric Parts, in Proc of the COMPCONTROL'77 International Conference, Warsaw, Poland, pp. 194-202, 1977

[32] Stark, J.: Product Lifecycle Management: $21^{\text {st }}$ Century Paradigm for Product Realisation, Birkhäuser, Heidelberg, 2004

[33] I. Cser, M. Juhász, K. Szabó, L. Horváth (I), L. Horváth (II), L. Tolnai: Computer-aided Planning of Machining Process. Preprints of the fifth international conference PROLAMAT'82, organized by IFIP and IFAC, Leningrad (now Saint Petersburg), 1982

[34] R. Viruez, S. Machado, L. M. Zamarreno, G. León, F. Beaude, S. Petitrenaud, J.-B. Heyberger: A Tool to ease Modelica-based Dynamic Power System Simulations, in proc of the $12^{\text {th }}$ International Modelica Conference, Prague, Czech Republic, pp. 235-239, 2017

[35] Ackoff, R. L.: From Data to Wisdom, Journal of Applies Systems Analysis, Vol. 16, No 1, pp. 3-9, 1989

[36] L. Horváth, I. J. Rudas: Multifunctional Engineering Space Supports Integration in Content Based Modeling, in proc. of the $11^{\text {th }}$ IEEE International Symposium on Applied Computational Intelligence and Informatics, Timisoara, Romania, pp. 51-56, 2016 